Sonja R. Vossenberg-Postma

Ytje T. Sikkema

Ioana Drogt-Bilaseschi

Nynke A. Bruins-Lange

Corine M. de Jager

Theo van Maaren

Veronica van der Pol

E. Christiaan Boerma

\section{Direct transfer of long-stay ICU patients to a nursing-home rehabilitation unit: focus on functional dependency}

Accepted: 10 August 2015

Published online: 26 August 2015

(C) The Author(s) 2015. This article is published with open access at Springerlink.com

Electronic supplementary material The online version of this article (doi:10.1007/s00134-015-4029-1) contains supplementary material, which is available to authorized users.

Dear Editor,

There is growing awareness of the potential challenges that may be associated with life after ICU survival. Post-ICU hospital readmission, long-lasting functional disability and cognitive impairment have been reported and are associated with reduction in quality of life and substantial utilisation of healthcare resources $[1,2]$. The impact of such post-ICU sequelae urges us to develop smart strategies for post-ICU rehabilitation, aiming for an immediate and continuous process of physical and psychological stimulation of individual ICU patients, beyond physical and virtual institutional walls.

An essential component in this chain of events is the transfer from the ICU to the next care facility. Traditionally ICU patients are referred to a hospital ward. However, bridging the gap between the ICU and hospital wards has been cumbersome in long-stay ICU patients. Despite the widespread introduction of a large variety of step-down units, its results are far from unequivocal; for individual patients the gap remains unbridgeable, fuelling additional initiatives, including rapid response teams and liaison nurse services [3]. However, the majority of these interventions are technology-centred, system-oriented rather than patientoriented, and none of these units are (additionally) equipped for rehabilitation.

Nursing-home rehabilitation units (NRU) traditionally incorporate multidisciplinary assessment of functional dependency, including physical, psychological and social deficits. A simultaneous goal setting for a variety of functional domains will be formulated, as a basis for tailor-made coordinated rehabilitation [4]. We hypothesized that, despite limitations in medico-technical support, direct step-down to an NRU could be a safe and suitable alternative for a specific group of severely disabled ICU patients.

To this end we performed a singlecentre before-after study. Patients with an ICU length of stay (LOS) longer than $48 \mathrm{~h}$, a hospital ward as first post-ICU step-down unit and an
NRU as final destination were selected (indirect transfer group, ITG). In the second phase, after an intervention to redirect patient flow, patients with an ICU LOS longer than $48 \mathrm{~h}$ and a direct transfer to an NRU were prospectively included (direct transfer group, DTG). Baseline characteristics, LOS in subsequent treatment facilities, readmission rate, final discharge status and consecutive Barthel indices (BI) were recorded [5]. Nonparametric tests were applicable.

In a 5-year period we included 31 patients in the ITG and 24 patients in the DTG, representing $0.7 \%$ of the total ICU population. At ICU discharge BI did not differ between groups [data are presented as median (IQR); ITG 2 (0-2) vs. DTG 2 (1-6), $p=0.15]$. Severity of illness, days of mechanical ventilation and LOS ICU were significantly higher in the DTG (ESM). Primary endpoint, the BI at NRU discharge, was not different between groups [ITG 16 (14-18) vs. DTG 16 (11-18), $p=0.50$; Fig. 1]. However, LOS post ICU was significantly longer in the ITG [ITG 74 (58-106) vs. DTG 46 (19-117), $p=0.03$; Fig. 1]. In addition, hospital readmission rates, mortality and final discharge status did not differ between groups (ESM).

Our data suggest that an NRU as the first post-ICU step-down facility in functionally dependent patients

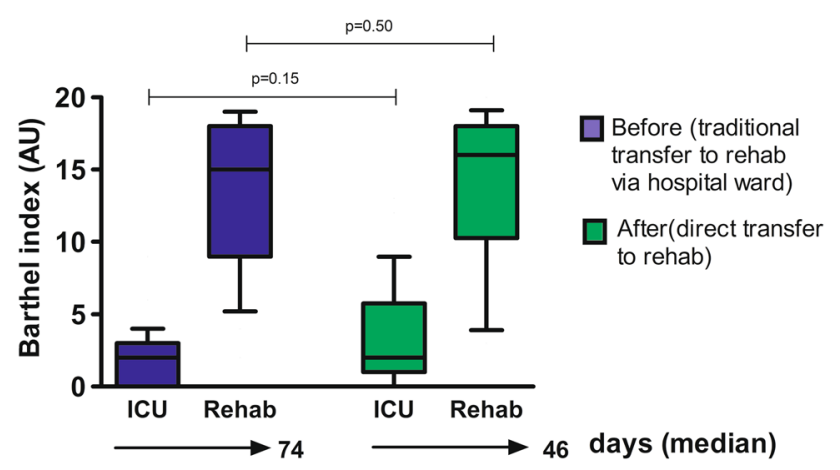

Fig. 1 Functional dependency at discharge ICU and rehab before and after intervention in the step-down destination post ICU 
may be associated with equal functional outcome in a shorter time frame, as compared to the traditional pathway. We hope this may fuel initiatives to focus on functional rather than medico-technical support in this specific group of long-stay ICU patients.

\section{Compliance with ethical standards}

Conflicts of interest The authors declare that they have no conflict of interest.

Ethics Because of the descriptive beforeafter setting of the study, institutional review board approval, informed consent and registration in a public trial register were considered inapplicable.

Open Access This article is distributed under the terms of the Creative Commons Attribution-NonCommercial 4.0 International License (http://creativecommons.org/ licenses/by-nc/4.0/), which permits any noncommercial use, distribution, and reproduction in any medium, provided you give appropriate credit to the original author(s) and the source, provide a link to the Creative Commons license, and indicate if changes were made.

\section{References}

1. Herridge MS, Tansey CM, Matté A et al (2011) Functional disability 5 years after acute respiratory distress syndrome. N Engl J Med 364:1293-1304. doi: 10.1056/NEJMoa1011802
2. Kahn JM, Benson NM, Appleby D, Carson SS, Iwashyna TJ (2010) Longterm acute care hospital utilization after critical illness. JAMA 303:2253-2259. doi:10.1001/jama.2010.761

3. Green A, Edmonds L (2004) Bridging the gap between the intensive care unit and general wards - the ICU liaison nurse. Intensive Crit Care Nurs 20:133-143. doi: 10.1016/j.iccn.2004.02.007

4. Rubenstein LZ, Stuck AE, Siu AL, Wieland D (1991) Impacts of geriatric evaluation and management programs on defined outcomes: overview of the evidence. J Am Geriatr Soc 39:8S-16S; discussion $17 \mathrm{~S}$

5. Collin C, Wade DT, Davies S, Horne V (1988) The Barthel ADL index: a reliability study. Int Disabil Stud 10:61-63
S. R. Vossenberg-Postma .

T. van Maaren - V. van der Pol

Elderly Care Medicine, Zorggroep

Noorderbreedte, Leeuwarden,

The Netherlands

\section{Y. T. Sikkema}

Department of Emergency Medicine, Medical Centre Leeuwarden, Leeuwarden, The Netherlands

I. Drogt-Bilaseschi - N. A. Bruins-Lange C. M. de Jager · E. C. Boerma (®) Department of Intensive Care, Medical Centre Leeuwarden, P.O. Box 888, 8901BR Leeuwarden, The Netherlands e-mail: e.boerma@chello.nl Tel.: +31582866737 